\title{
Multibit Memory Cells Based on Spin-Orbit Torque Driven Magnetization Switching of Nanomagnets with Configurational Anisotropy
}

\author{
Shaik Wasef, Selma Amara, Meshal Alawein, and Hossein Fariborzi* \\ CEMSE Division, King Abdullah University of Science and Technology (KAUST) \\ *Email - hossein.fariborzi@kaust.edu.sa
}

\begin{abstract}
In this work, we report the fabrication and characterization of novel four and six terminal current-driven magnetic memory cells. In particular, we experimentally demonstrate the magnetization switching of triangular and square magnets through spin-orbit torque by in-plane currents in a $\mathrm{Pt} / \mathrm{Ni}_{81} \mathrm{Fe}_{19}(\mathrm{Py})$ heterostructure. The spin torques, generated by applying a constant current in one of multiple Pt wires, are used to switch a Py film between its multiple stable magnetic states, as quantified by anisotropic magnetoresistance (AMR) and tunnel magnetoresistance (TMR) measurements at room temperature. The observations have also been confirmed by micromagnetic simulations.

(Keywords: configurational anisotropy, spin orbit torque, spin transfer torque, MTJ)
\end{abstract}

\section{Introduction}

For many decades, the electronic community has relied on Moore's law as the guideline for its technology roadmap. As we approach the $7 \mathrm{~nm}$ CMOS technology node and beyond, we are confronted with serious challenges that might diminish the benefits of further scaling. This has led to an extensive search for a next-generation computing and memory device. Spintronics is an emerging field in which the elusive quantum attribute, known as "spin", is used as a (noncharge) state variable. Although the field is relatively new, it has revolutionized data storage and may enable novel quantum computing applications and functional scaling beyond current technology node.

Magnetic random-access memory (MRAM) is a spintronic memory technology that stores data in the alignment of spins in the ferromagnet comprising the magnetic tunnel junction (MTJ). Although the current MRAM technology shows the potential to be used as a "universal memory", it still suffers from high cost of fabrication and low density of bits.

In order to increase the memory density, one can use the fact that the anisotropy of magnets can be tailored by material and shape engineering. For example, imposing symmetry on the magnet and the introduction of certain defects have been shown to lead to interesting effects such as configurational anisotropy [1], and nucleating centers [2]. Configurational anisotropy, which is a high order form of shape anisotropy, leads to the introduction of multiple easy axes in some magnetic materials of special shapes (e.g. triangle, square, star, etc.). Recently, it has been shown that a Py nanomagnet in the shape of a triangle show triaxial anisotropy [1]. In this work, we use these observations and present multibit memory cells based on shape engineering of Py films where information is written through spinorbit torque interactions in Pt wires.

\section{Multibit Memory Cell}

The proposed memory cell is comprised of an MTJ (of square or triangular shape) in the center of several Pt wires, where the number of wires depends on the number of states encoded (e.g. a four-state memory will require two Pt wires), as shown in Fig. 1. In Fig. 1a, we placed a square multilayer stack (tilted $45^{\circ}$ ) in the center of two perpendicular wires, and in Fig. $1 \mathrm{~b}$ we placed a triangular stack in the center of three wires. Because of the spin Hall effect [3], when a charge current passes through a Pt wire, electrons of opposite spin scatter in opposite directions. The diffusion of accumulated spins generates a transverse (pure) spin current that can switch the local magnetization of the free Py layer between its multiple stable states. 


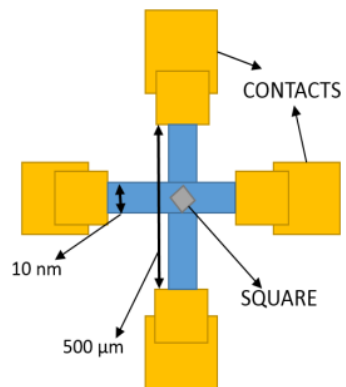

(a)

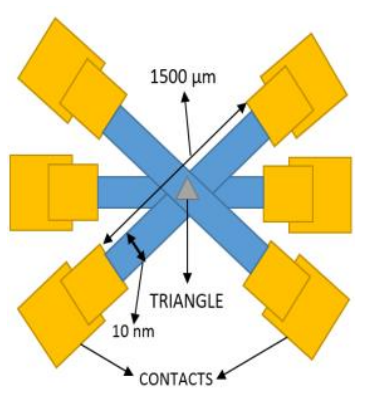

(b)
Fig. 1: Top view of the layout without top contact for (a) square and (b) triangular magnets.

\section{Experimental Setup and Results}

The multilayer stack we fabricated is Pt (25 $\mathrm{nm}) / \mathrm{Py}(3 \mathrm{~nm}) / \mathrm{MgO}(1 \mathrm{~nm}) / \mathrm{Py}(12 \mathrm{~nm}) / \mathrm{Pt}(25 \mathrm{~nm})$. As shown in Fig. 2, electrical contacts of $\mathrm{Ti}$ $(10 \mathrm{~nm}) / \mathrm{Au}(100 \mathrm{~nm})$ are placed over each arm in the structure.

The resistance of the multilayer stack depends on the angle $(\beta)$ between the top Py $(12 \mathrm{~nm})$ pinned layer and the bottom Py (3nm) free layer. A current of $20 \mathrm{~mA}$ between the contacts 1 and 2 is used to switch the magnetization in the free layer, then a reading current of $14 \mu \mathrm{A}$ is passed between contact 3 and contact 2 to measure the resistance of the MTJ. The resistance of the multilayer is given by the following equation [4]

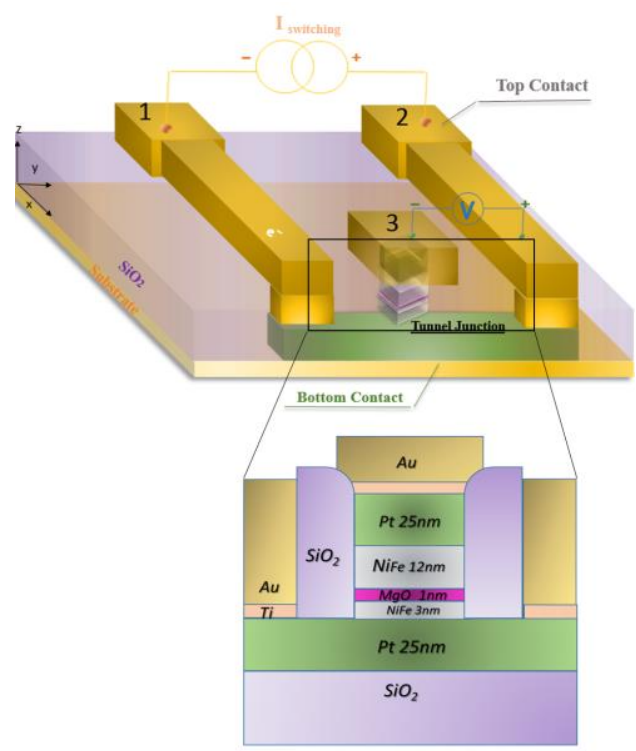

Fig. 2: Schematic representation of the device with the junction stack details.

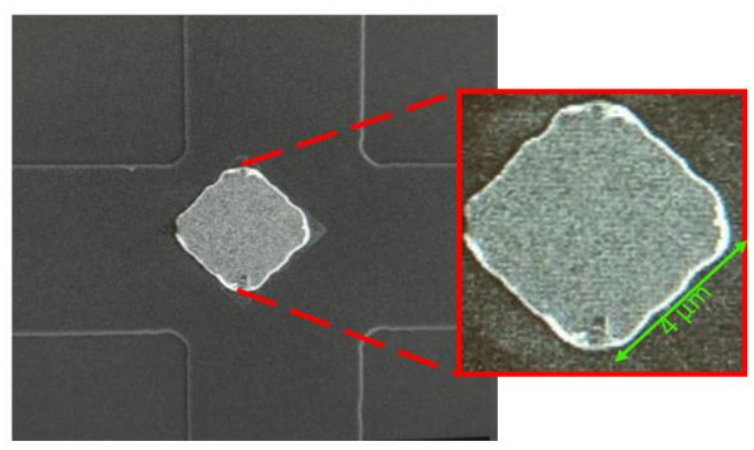

(a)

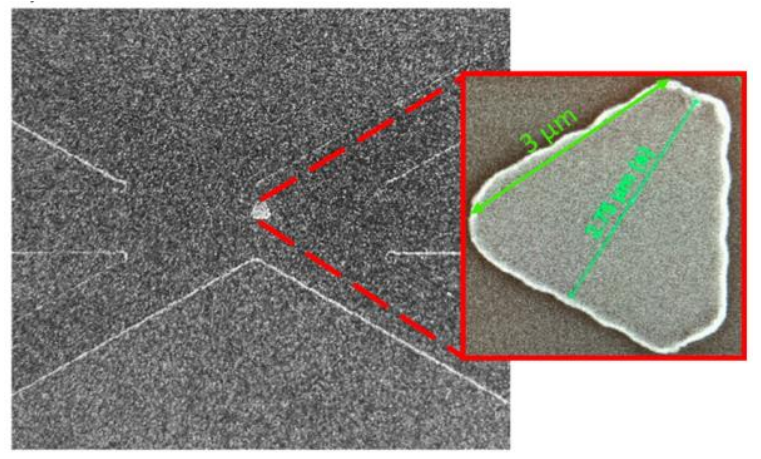

(b)

Fig. 3: SEM image of (a) square and (b) triangle magnetic memory structures

$$
R(\beta)=R_{a v}-1 / 2(\Delta R) \cos (\beta)
$$

where $R_{a v}=1 / 2\left(R_{a p}+R_{p}\right)$ and $\Delta R=R_{a p}-R_{p}$. Here $R_{p}$ and $R_{a p}$ are the resistances in the parallel and antiparallel state, respectively. As per Eq. (1), for a square structure, apart from $R_{a p}$ and $R_{p}$, we measure two equal resistances $\mathrm{R}_{90^{\circ}}$ and $\mathrm{R}_{270^{\circ}}$ corresponding to the perpendicular orientations of the magnetization in the free layer with respect to the pinned layer. Similarly for a triangle, apart from $R_{a p}$ and $R_{p}$, we measure four resistances $\mathrm{R}_{60^{\circ}}, \mathrm{R}_{120^{\circ}}, \mathrm{R}_{240^{\circ}}$ and $\mathrm{R}_{300^{\circ}}$. However, because of the structure symmetry, $\mathrm{R}_{60^{\circ}}=\mathrm{R}_{300^{\circ}}$, and $R_{120^{\circ}}=R_{240^{\circ}}$. Fig. 4 shows the stable states measured for square and triangular structures. We note that in order to break the symmetry to create four and six state devices, one can also introduce an ellipsoidal pinned layer with an easy axis appropriately tilted relative to the easy axis of the free layer. 


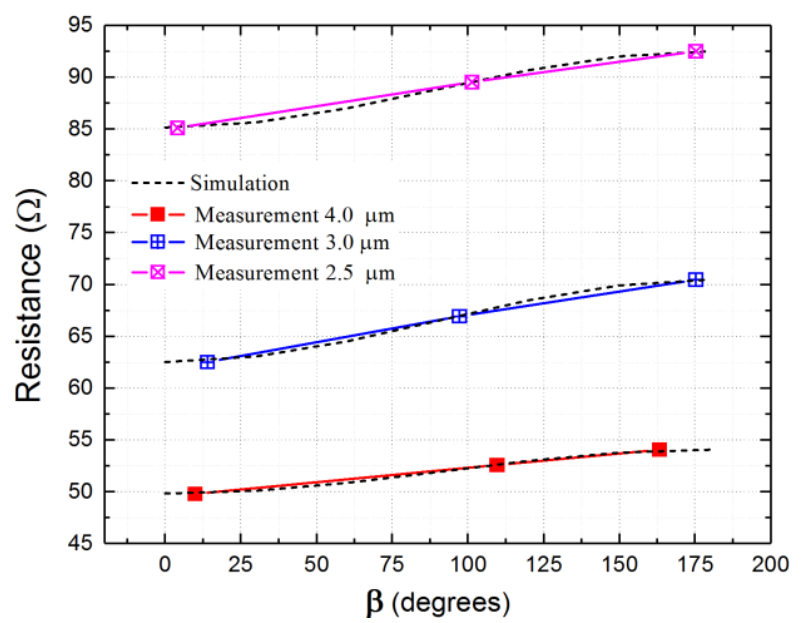

(a)

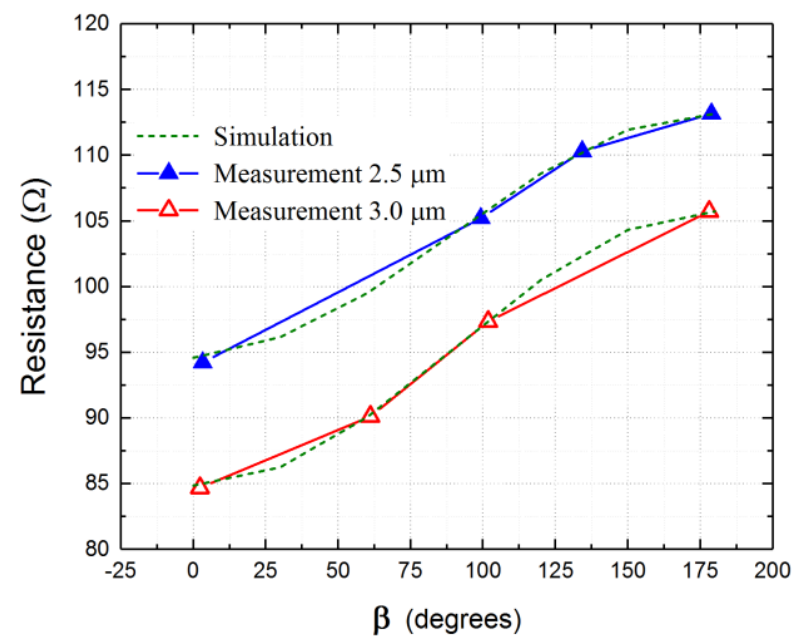

(b)

Fig. 4: Representation of (a) 3 states for squares and (b) 4 states for triangles.

In addition to our experimental results, we have performed micro magnetic simulations on square and triangular magnets using the software package OOMMF [2]. In Fig. 5a the alignment of the magnetic vectors along the diagnols of the square confim the existance of biaxial anisotropy in square Py for some appropriate physical dimension. Similiarly for a triangle we observed three easy axes (triaxial anisotropy).

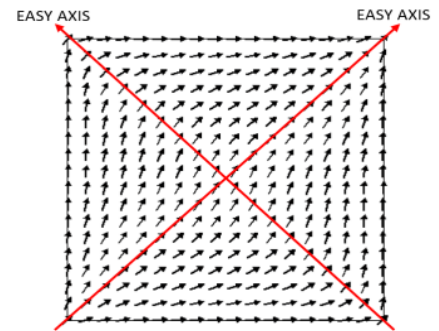

(a)

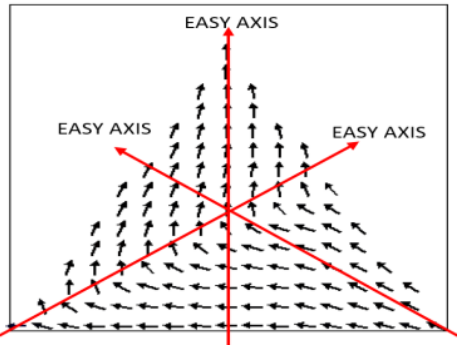

(b)

Fig. 5: Schematics showing magnetic simulation of (a) square (b) triangle.

\section{Conclusion}

In this paper, we have demonstrated multi-state magnetic storage devices with spin-orbit torque as the writing mechanism. With the observed results, one can envisage novel multibit memory cells that would help in the further miniaturization and manufacturing cost reduction of memory arrays.

\section{References}

[1] D. E. Endean, C. T. Weigelt, R. H. Victora, and E. D. Dahlberg, Applied Physics Letters, Vol. 103, No. 4, pp. 042409 (2013).

[2] D. Bhowmik, L. You, and S. Salahuddin, Nature Nanotechnology, Vol. 9, No. 1, pp. 59-63 (2014).

[3] D. Bhowmik, L. You, and S. Salahuddin, Nature Nanotechnology, Vol. 9, No. 1, pp. 59-63 (2014).

[4] Telepinsky, Yevgeniy, et al. "Towards a sixstate magnetic memory element." Applied Physics Letters 108.18 (2016): 182401.

[5] OOMMF: National Institute of Standard and Technology 2002. 